\title{
Surgical Repair of the Anomalous Origin of the Right Pulmonary Artery From the Ascending Aorta
}

\author{
Miguel Arboleda, Iván Niño de Guzman, Eva Ticona, Gabriela Morales, Edgard Gloria, \\ Plinio Obregon, Alfredo Lora, María Ganiku, Manuel Adrianzen, Liana Falcon \\ Lima - Peru
}

\begin{abstract}
The anomalous origin of the right pulmonary artery (AORPA) from the ascending aorta is a rare congenital malformation. We describe an infant who underwent a surgical correction with direct anastomosis between the right pulmonary artery and the pulmonary trunk. Eighteen months later, the patient remains asymptomatic, and no significant residual stenosis was detected on angioresonance.
\end{abstract}

The aortic origin of the right or left pulmonary artery is an infrequent cardiac anomaly whose incidence is $<1 \%$ of all the congenital cardiac malformations.

This cardiopathy consists of the abnormal origin of one of the pulmonary arteries directly from the aorta, either the ascending portion or the transverse aortic arch.

In $70-80 \%$ of cases, it is the right pulmonary artery (RPA) that grows directly from the ascending aorta, and in $75 \%$ of cases this cardiopathy is associated with patent ductus arteriosis (PDA).

The clinical setting is characterized by increased pulmonary blood flow, congestive heart failure, and cyanosis when the pulmonary pressure and vascular resistance are too elevated.

The diagnosis must be made by echocardiography. The exclusion of one lung from the pulmonary radionucleotide angiography injected through a peripherial vein is a strong indicator of this diagnosis. Nevertheless, angiocardiography offers very important additional information ${ }^{1}$.

The corrective surgical treatment is unquestionable and urgent and must be performed early after diagnosis because without it mortality rates reach $70 \%$ before 6 months of age ${ }^{2}$.

We describe the case of a nursing female infant with such an anomaly illustrated by echocardiographic and angiocardiographic imaging performed before the surgical correction and current ones obtained by magnetic resonance imaging (MRI) 9 months after the surgery.

\section{Case Report}

A 6-month-old female patient with a background of pneumonia was diagnosed by echocardiography as having PDA with important

Instituto Nacional del Corazón - Departamento de Cardiopediatría (ESSALUD), Lima - Perú and Resonancia Magnética S.A. (RESOMASA), Lima - Perú Mailing address: Miguel Arboleda. Calle Trípoli, 350 - dpto. 301 - Miraflores - Lima 18 - Lima - Peru - E-mail: miguel_arboleda@hotmail.com Received for publication in $04 / 14 / 2003$

Accept in 07/21/2004 hemodynamic distress. At 5 months of age, the infant underwent surgery to close the PDA, still experiencing pulmonary plethora, severe pulmonary hypertension, episodes of cyanosis, and intrahospital respiratory infection. The study with radionucleotide angiography showed perfusional exclusion of the right lung even though a chest X-ray showed a bilateral accentuated pulmonary vasculature pattern and signs of pulmonary hypertension. A new echocardiography detected the anomalous origin of the right pulmonary artery (AORPA), the absence of residual PDA, and severe pulmonary hypertension. Subsequently, the patient underwent cardiac catheterization that confirmed the AORPA from the ascending aorta and severe bilateral pulmonary hypertension, being suprasystemic on the left side.

When the infant was 6 months of age and weighed 5,150 grams, the surgical repair was performed with the help of extracorporeal circulation with hypothermia of $28^{\circ} \mathrm{C}$ and cold blood cardioplegia. The RPA arose from the left posterior wall of the ascending aorta, far enough from the aortic valve plane and from the origin of the brachiocephalic trunk.

The previously ligated PDA was excised from the aorta and main pulmonary artery. Both pulmonary arteries were widely dissected to obtain their maximum mobilization. With the heart arrested, the RPA of the aorta was disconnected, repairing the opening with a continuous polipropilene suture. Next, a longitudinal incision was made on the lateral wall of the pulmonary trunk where the RPA was anastomosed in termino-lateral fashion from behind the aorta and without the use of prosthetic material.

At the conclusion of the surgery, the pressure on the main pulmonary artery was approximately $50 \%$ of the systemic pressure. The girl was weaned off ventilation assistance after 24 hours, progressed satisfactorily, and was released 7 days after surgery. Eighteen months after surgery, the patient is asymptomatic without medication and in good general condition.

\section{Discussion}

The aortic origin of a pulmonary artery is an infrequent anomaly, being $<1 \%$ of all congenital cardiopathies. Although either one or both of the pulmonary arteries can originate directly from the aorta, either from the ascending portion or the transverse arch portion, in almost $80 \%$ of cases it is the RPA that originates from the ascending aorta and in approximately $75 \%$ of cases a PDA is present ${ }^{1,2}$. Nevertheless, other cardiac anomalies can coexist, amongst which Fallot's tetralogy is the most frequent ${ }^{3,4}$. 
Commonly, the clinical episode is dominated by accentuated pulmonary vasculature and severe early pulmonary hypertension that leads to congestive heart failure and crying-triggered cyanosis. Recurrent respiratory infections frequently aggravate the clinical status.

Nonetheless, the low incidence of this anomaly can lead to confusion with other more frequent conditions with very similar clinical episodes. Such is the case described herein, the first reported in our country, which initially was not detected with the clinical examination and the first echocardiographic test.

Certain clinical signs and data from supplementary examinations exist and without being specific are very frequent in this anomaly. The presence of cyanosis on effort can be explained by a right to left shunt through the PDA or from the persistence of the foramen ovale in the presence of high pulmonary resistance.

The injection of radioactive isotopes through a peripheral vein shows perfusion exclusion of the right lung when it is the right artery that originates from the ascending aorta. This finding can lead us to erroneous interpretations, such as thrombosis or accidental ligature of the RPA if a surgical procedure had been previously conducted.

The echocardiography is usually sufficient for an accurate diagnosis ${ }^{5}$. The images obtained from parasternal short axis and apical 4-chamber images allow a precise diagnosis (fig. 1).

Nevertheless, the cardiac catheterization can provide important anatomic and presoric details (fig. 2). It is important to point out the elevated pulmonary pressure that reaches suprasystemic levels in the normally perfused lung, in this case the left one due to receiving all systemic venous return. Lastly, the catheterization must be performed in case of diagnostic doubt.

Less invasive and with optimum results, magnetic angioresonance is a good diagnostic alternative ${ }^{6}$.

Although the first references to this pathology date back to the XIX century, in Fraentzel's 1868 historic reference, most of the publications report a small number of cases, one of the longest series being that published by Abu-Sulaiman et al ${ }^{7}$ with 14 patients operated on throughout a period of 36 years.

Given the seriousness of this anomaly, surgical repair must be performed as early as possible; nevertheless, there are reports of successful interventions even in adolescent patients ${ }^{8}$. Although corrective surgery can almost always be performed, the banding of the anomalous pulmonary artery and the ligature of the PDA have been described as a palliative alternative in special cases such as premature infants with very low weight ${ }^{9}$.

Several surgical strategies have been proposed, such as deep hypothermia and total circulatory arrest ${ }^{2}$, use of vascular grafts, and placement of the RPA in front of the aorta.

In the presented case, extracorporeal circulation and hypothermia at $28^{\circ} \mathrm{C}$ with cold blood intermittent cardioplegia was utilized. As reported in most publications, it was possible to reestablish the connection between the RPA and the pulmonary trunk from behind the aorta without the use of grafts and by direct anastomoses of both structures. Likewise, the posterior wall of the aorta was directly repaired with continuous suture.

Hospital mortality in the most serious series of patients operated on ranged between 21 and $25 \%{ }^{7,10}$. No late postoperative deaths were reported, a fact that suggests that survival after surgical repair is satisfactory. The patient presented in this clinical case had a satisfactory postoperative evolution with a quick and significant reduction in pulmonary pressure and was released 7 days after the surgery.
Eighteen months after surgery, the patient is asymptomatic and in excellent general health. A control echocardiography shows normal-sized cardiac cavities, normal pulmonary pressure, and a pressure gradient of $12 \mathrm{mmHg}$ between the main and the right pulmonary arteries. The MRI showed a normal blood flow towards both lungs and the absence of stenosis of the main pulmonary artery and its branches.

Nevertheless, a long-term follow-up is required because the most frequent residual lesion is the stenosis of the pulmonary branch at the level of the anastomosis. Such a condition requires some type of intervention, and in close to $50 \%$ of the cases a repetition of the surgical repairs, balloon dilation or placement of stents is needed ${ }^{7}$.

In conclusion, the anomalous origin of the right pulmonary artery from the ascending aorta is a very infrequent congenital anomaly, but with a bad prognosis if not promptly treated; it must always be considered to increase the chances of early detection and correction. The corrective surgical treatment is almost always possible and the result satisfactory. The residual stenosis of the pulmonary branch is frequent but can be treated by interventional catheterization with low risk and in an elective manner.

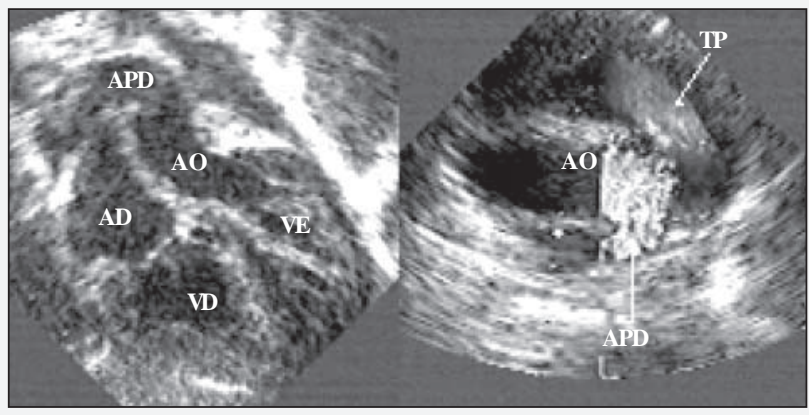

Fig. 1 - Echocardiogram: on the left the apical 4-chamber cut showing the right pulmonary artery (RPA) derived directly from the ascending aorta; on the right, with the short axis parasternal cut, the pulmonary trunk (TP) anteriorly and the RPA originating from the aorta.

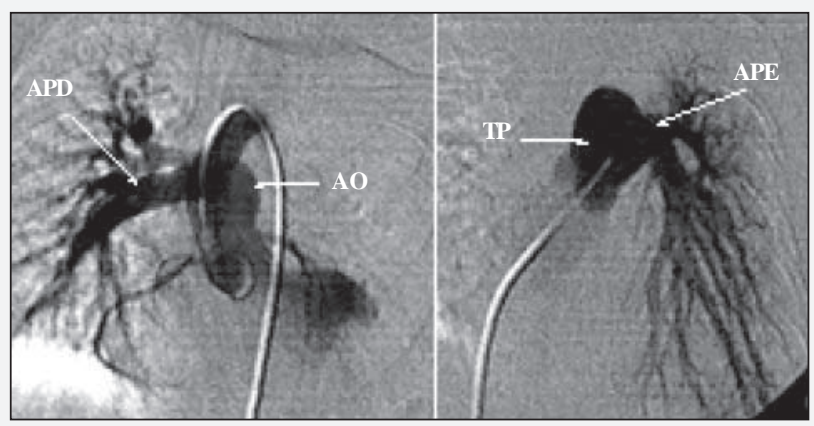

Fig. 2 - Angiography: on the left, the right pulmonary artery originating directly from the ascending aorta (AO); to the right, the pulmonary trunk (PT) flowing directly into the left pulmonary artery with total absence of the right pulmonary artery.

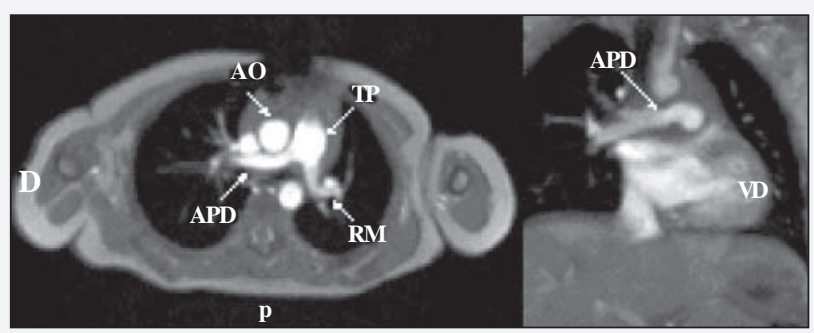

Fig. 3 - Angioresonance: postoperative view of the surgical correction showing normal blood flow through the pulmonary trunk (TP) and both right and left pulmonary arteries (RPD, RPI). 


\section{References}

1. Kirklin J, Barratt-Boyes B. Origin of the right or left pulmonary artery from the ascending aorta. En : Cardiac Surgery. New York: John Wiley \& Sons, 1986; 939-44.

2. Malo P, Pérez V. Origen aórtico de un rama de la arteria pulmonar. En: Sánchez P. Cardiología Pediátrica.Clínica y Cirugía. Barcelona: Salvat Editores. 1986; 346-51.

3. Amaral F, Teixeira M, Granzotti J, Manso P, Vicente W. Anomalous origin of the left pulmonary artery from the ascending aorta. Successful surgical correction in an infant with Fallot's tetralogy. Arq Bras Cardiol. 2002; 79:541-3.

4. Rivera IR, Moises VA, Silva CC, et al. Anomalous origin of the right pulmonary artery from the ascending aorta (Hemitruncus). Arq Bras Cardiol. 1998; 70: 341-4.

5. Girona J, Casaldaliga J, Sánchez C, Yeste D, Miro L. Anomlous origin of the right pulmonary artery from the ascending aorta. Its echo-Doppler diagnosis. Rev Esp Cardiol. 1993; 46:260-2.

6. Kim TK, Choe YH, Kim HS, et al. Anomalous origin of the right pulmonary artery from the acending aorta: diagnosis by magnetic resonance imaging. Cardiovasc Intervent Radiol. 1995; 18: 118-21.

7. Abu-Sulaiman RM, Hashmi A, McCrindle BW, Williams WG, Freedom RM. Anomalous origin of one pulmonary artery from the ascending aorta: 36 years' experience from one centre. Cardiol Young. 1998; 8:449-54.

8. Kuinose M, Tanemoto K, Murakami T, Kanaoka Y, Kobayashi G, Makabe M. Surgical treatment for a 16-year-old girl with anomalous origin of the right pulmonary artery from ascending aorta. Jpn J Thorac Cardiovasc Surg. 1998; 46:380-4.

9. Marui A, Mochizuki T, Mitsui N, Koyama T, Horibe M. Anomalous origin of the right pulmonary artery from the ascending aorta - a report of three operative cases. Nippon Kyobu Geka Gakkai Zasshi. 1997; 45: 1189-94.

10. Nonoyama M, Imai Y, Sawatari K, Nagatsu M. Anomalous origin of the right pulmonary artery from the ascending aorta: a report of eight cases. Nipón Kyobu Geka Zasshi. 1994; 42: 83-9. 